\title{
Multiband Circular Polarizer Based on Fission Transmission of Linearly Polarized Wave for $\boldsymbol{X}$-Band Applications
}

\author{
Farman Ali Mangi, ${ }^{1}$ Shaoqiu Xiao, ${ }^{1}$ Ghulam Ali Mallah, ${ }^{2}$ Deedar Ali Jamro, \\ Imran Memon, ${ }^{3}$ and Ghulam Fatima Kakepoto ${ }^{1}$ \\ ${ }^{1}$ School of Physical Electronics, University of Electronic Science and Technology of China, Chengdu, Sichuan 610054, China \\ ${ }^{2}$ Department Computer Science, Shah Abdul Latif University Khairpur, Sindh 66020, Pakistan \\ ${ }^{3}$ College of Computer Science, Zhejiang University, Hangzhou, Zhejiang 310027, China
}

Correspondence should be addressed to Farman Ali Mangi; farmanali29@yahoo.com

Received 2 June 2016; Accepted 11 July 2016

Academic Editor: Jit S. Mandeep

Copyright (C) 2016 Farman Ali Mangi et al. This is an open access article distributed under the Creative Commons Attribution License, which permits unrestricted use, distribution, and reproduction in any medium, provided the original work is properly cited.

A multiband circular polarizer based on fission transmission of linearly polarized wave for $x$-band application is proposed, which is constructed of $2 \times 2$ metallic strips array. The linear-to-circular polarization conversion is obtained by decomposing the linearly incident $x$-polarized wave into two orthogonal vector components of equal amplitude and $90^{\circ}$ phase difference between them. The innovative approach of "fission transmission of linear-to-circular polarized wave" is firstly introduced to obtain giant circular dichroism based on decomposition of orthogonal vector components through the structure. It means that the incident linearly polarized wave is converted into two orthogonal components through lower printed metallic strips layer and two transmitted waves impinge on the upper printed strips layer to convert into four orthogonal vector components at the end of structure. This projection and transmission sequence of orthogonal components sustain the chain transmission of electromagnetic wave and can achieve giant circular dichroism. Theoretical analysis and microwave experiments are presented to validate the performance of the structure. The measured results are in good agreement with simulation results. In addition, the proposed circular polarizer exhibits the optimal performance with respect to the normal incidence. The right handed circularly polarized wave is emitted ranging from $10.08 \mathrm{GHz}$ to $10.53 \mathrm{GHz}$ and $10.78 \mathrm{GHz}$ to $11.12 \mathrm{GHz}$, while the left handed circular polarized wave is excited at $10.54 \mathrm{GHz}-10.70 \mathrm{GHz}$ and $11.13 \mathrm{GHz}-11.14 \mathrm{GHz}$, respectively.

\section{Introduction}

Polarization is an important property of electromagnetic (EM) waves due to inherent polarization sensitivity of materials. It is widely used in various electromagnetic wave applications including radar and wireless communication system. Circular polarizer can transfer linearly polarized wave into circularly polarized wave under the normal incidence of plane wave. According to existing research, circular polarizer can be designed by employing photonic $[1,2]$, chiral structures [3-7], metasurface, metamaterials [8], meanderline [9], slots of different structures [10], waveguide [11], and grating structures [12].

Wideband circular polarizer is proposed using stacked dual-layer periodic arrays [10], which are separated by large distance of 0.33 operational wavelengths. The multiband of polarizer is useful for many applications. The polarization characteristics of transmitted wave of dual-band asymmetry chiral metamaterial structure were demonstrated. The proposed structure achieved RHCP and LHCP wave at four distinct resonant frequencies [13]. The multiband circular polarizer achieved the RHCP and LHCP wave at three distinct resonant frequencies by using incident polarized wave [14]. The circular dichroism was obtained across a narrow band at resonance frequencies. The polarization characteristics of transmitted wave of dual-band asymmetry chiral metamaterial structures are demonstrated in [15]. Chiral metamaterials achieve electromagnetic wave properties, such as strong circular dichroism effect $[16,17]$ and giant optical activity [18-21]. 
In this paper, multiband circular polarizer based on fission transmission of linearly polarized wave for $x$-band application is proposed which is constructed of double layer periodic metallic strips array printed on both sides of dielectric structure. Firstly, this novel approach of "fission transmission of electromagnetic waves" is introduced to obtain giant circular dichroism, which means that the incident linearly polarized wave is converted into two orthogonal components through lower printed metallic strips layer. Simultaneously, the two transmitted orthogonal components impinge on the upper printed strips along $+z$ direction and converted into four orthogonal vector components at the end of structure. The $x$-linearly polarized incident wave decomposed into two orthogonal vector components $E_{x}$ and $E_{y}$ from lower printed metallic strips. Meantime, the transmitted $E_{x}$ and $E_{y}$ waves impinge on the top surface of structure along $+z$ direction to generate two pairs of orthogonal components from top layer of structure. Incidence and transmission of $E_{x}$ and $E_{y}$ through layer-by-layer sustain chain transmission of EM waves to achieve giant circular polarization at the other side of structure.

The proposed structure demonstrates the co- and crosspolarization transmission to realize circular polarization at distinct frequency bands. Firstly, a dual-band circular polarizer is introduced that converts linear circular polarized wave into right hand circular polarized wave with high conversion efficiency at $10.70 \mathrm{GHz}$ and $11.09 \mathrm{GHz}$. Subsequently, this concept is extended to compose a four-band circular polarizer and each band is broaden for circular polarized wave transmission. The proposed multiband circular polarizer achieves two orthogonal polarization eigenstates, right handed circular polarization (RHCP) wave at $10.08 \mathrm{GHz}-$ $10.53 \mathrm{GHz}$ and $10.78 \mathrm{GHz}-11.12 \mathrm{GHz}$ and left handed circular polarization state (LHCP) wave at $10.54 \mathrm{GHz}-10.70 \mathrm{GHz}$ and $11.13 \mathrm{GHz}-11.14 \mathrm{GHz}$, respectively.

The significant advantages of this proposed multiband circular polarizer have simple geometry and more broaden operating frequency bands comparing to reported designs [22-24], good circular polarization, easy fabrication, high conversion efficiency, and strong circular dichroism at operated frequency bands and can be used for the $x$-band microwave applications.

\section{Design Structure and Results}

2.1. Dual-Band Circular Polarizer. The pattern of unit cell is depicted in Figure 1. The two metallic strips with same size are printed on both sides of the substrate with different directions in XOY plane. The top printed strip is tilted at $45^{\circ}$ and the bottom strip is slanted at $60^{\circ}$ along $x$ - and $y$-axis, respectively. Initially, numerical simulation is performed to calculate the transmission behaviour of dual-band circular polarizer. The incident $x$-linearly polarized wave is used as excitation source along $+z$ direction. The model is excited by floquet port 1 with linearly polarized wave range from 8 to $12 \mathrm{GHz}$.

The geometric parameter of dual-band circular polarizer unit cell is denoted in Figure 1, that is, $t=0.787 \mathrm{~mm}, l=$ $10 \mathrm{~mm}, w=1.787 \mathrm{~mm}, p_{x}=15 \mathrm{~mm}$, and $p_{y}=15 \mathrm{~mm}$, respectively. The dielectric substrate Roger RT/duroid 5880

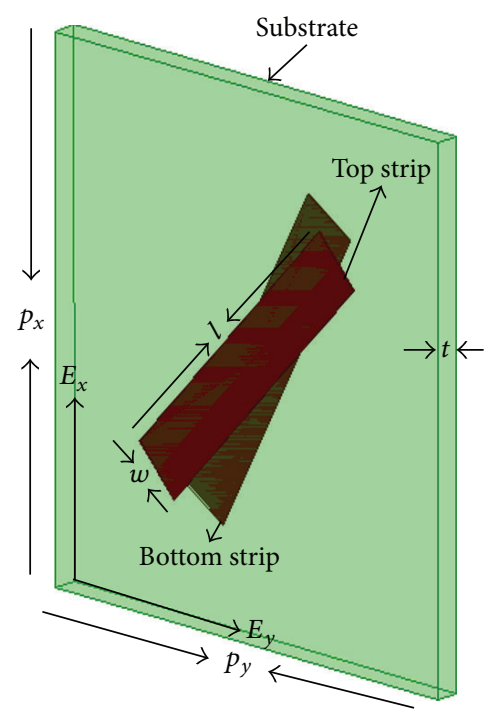

FIGURE 1: Schematic view of dual-band circular polarizer unit cell.

is employed with specifications are as follows: dielectric permittivity is $\mathscr{E} r=2.2$ with a loss tangent of 0.0009 . The ultrathin $(0.01 \mathrm{~mm})$ metallic strips are printed on both sides of substrate. The periodic boundary conditions, Master and Slave, are assigned to simulate the periodic geometrical structure along $x$ - and $y$-axis. The excitation of port 1 and port 2 is provided by floquet modes with two orthogonal waves which represent the vertically $\left(E_{x}\right)$ and horizontally $\left(E_{y}\right)$ polarized vector components of the incident wave, respectively. The $x$-linearly polarized wave is applied as excitation source through port- 1 along $+z$ direction.

Figure 2(a) presents the transmission coefficients of $T_{x x}$ and $T_{x y}$. The transmission coefficients of $T_{x x}$ are $5.69 \mathrm{~dB}$ $(f 1=10.70 \mathrm{GHz})$ and $6.48 \mathrm{~dB}(f 2=11.09 \mathrm{GHz})$, respectively. Meanwhile, the transmission coefficients of $T_{x y}$ are obtained, $2.90 \mathrm{~dB}(f 1)$ and $3.82 \mathrm{~dB}(f 2)$, respectively. In order to achieve polarization conversion of transmitted waves, the axial ratio $\mathrm{AR}=\left|T_{x y}\right| /\left|T_{x x}\right|$ and their phase differences $\varphi\left(\left|T_{x y}\right|\right)-\varphi\left(\left|T_{x x}\right|\right)$ of cross-polarization and copolarization transmissions are achieved for normal incidence of $x$ polarized wave.

Figure 2(b) shows the axial ratio between transmitted waves $T_{x x}$ and $T_{x y}$ which is equal to 1.3 at $10.70 \mathrm{GHz}$ (denoted as $f 1$ ) and 1.3 at $11.09 \mathrm{GHz}$ (denoted as $f 2$ ), and phase difference equals $89.68^{\circ}$ at $f 1$ and $91.56^{\circ}$ at $f 2$ as denoted in Figure 2(c). The axial ratio bandwidth of dual band is achieved ranging from $10.48 \mathrm{GHz}$ to $10.71 \mathrm{GHz}(\mathrm{BW})=2.3 \%$ and from $10.91 \mathrm{GHz}$ to $11.18 \mathrm{GHz}(\mathrm{BW})=2.7 \%$, as shown in Figure 2(b), respectively. The axial ratio bandwidth and transmission conversion efficiency at operated frequency bands $(f 1)$ and $(f 2)$ are comparatively better than reported work [14]. The RHCP waves is obtained at the operated frequency bands from $10.48 \mathrm{GHz}$ to $10.78 \mathrm{GHz}$ and $10.91 \mathrm{GHz}$ to $11.18 \mathrm{GHz}$.

Figure 2(b) shows the axial ratio between transmitted waves $T_{x x}$ and $T_{x y}$ which is equal to 1.3 at $10.70 \mathrm{GHz}$ (denoted as $f 1$ ) and 1.3 at $11.09 \mathrm{GHz}$ (denoted as $f 2$ ), and phase 


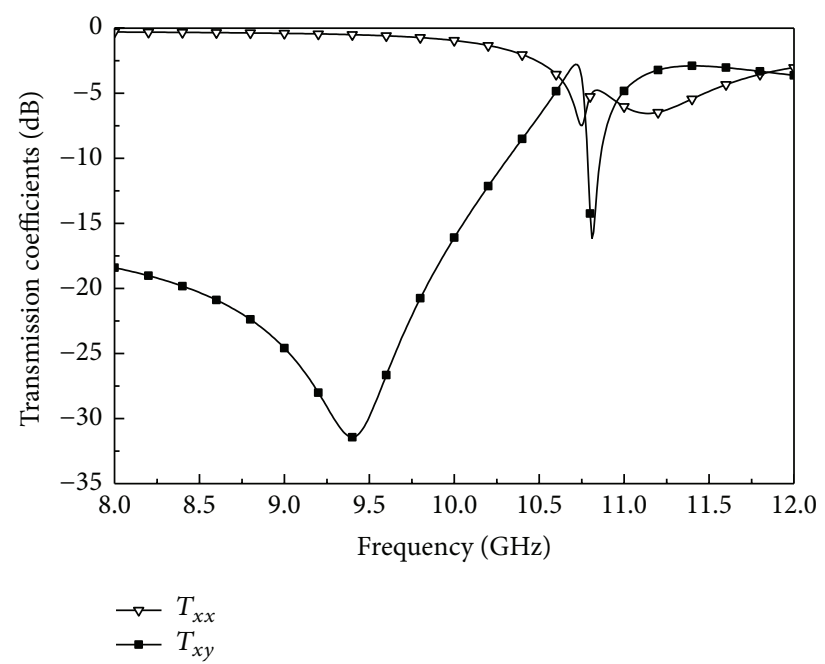

(a)

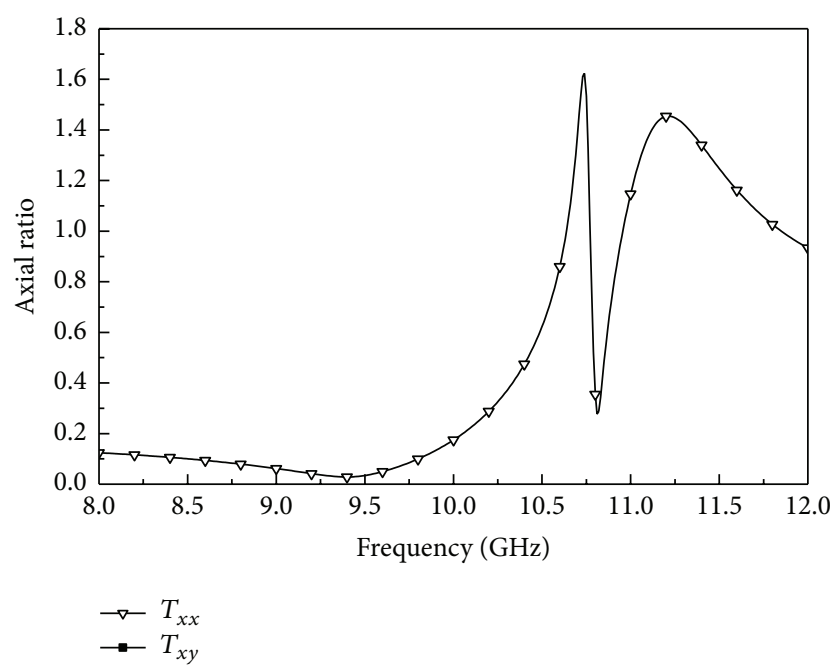

(b)

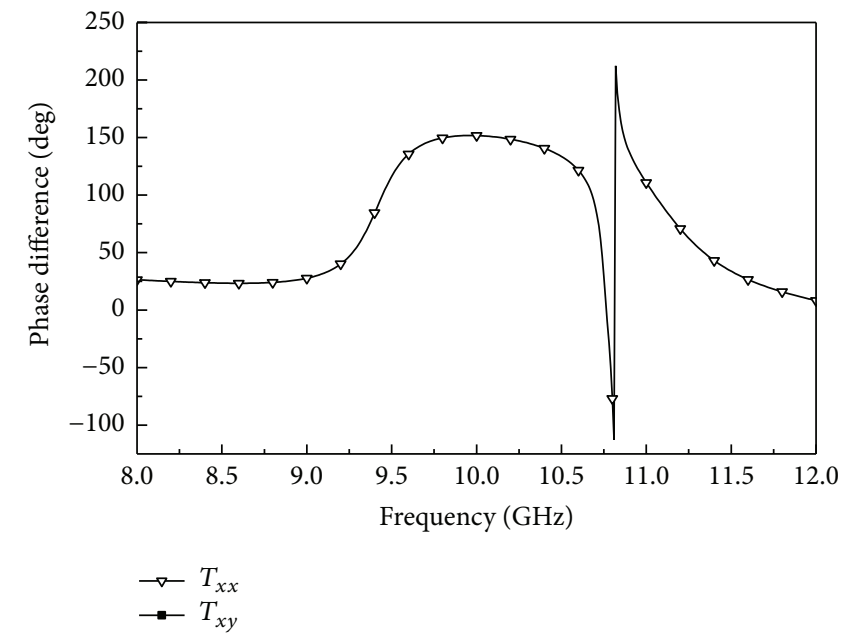

(c)

FIGURE 2: The figure presents the simulation results for dual-band circular polarizer. (a) shows the transmission coefficients of $T_{x x}$ and $T_{x y}$ versus frequency for normal incident wave at $\theta=0^{\circ}$, (b) represents the axial ratio of transmitted wave versus frequency, and (c) shows the phase difference between transmitted waves versus frequency of designed structure.

difference equals $89.68^{\circ}$ at $f 1$ and $91.56^{\circ}$ at $f 2$ as denoted in Figure 2(c). Figure 2(b) presents the dual-band ranging from $10.48 \mathrm{GHz}$ to $10.71 \mathrm{GHz}, \mathrm{BW}=2.3 \%$ and $10.91 \mathrm{GHz}$ to $11.18 \mathrm{GHz}, \mathrm{BW}=2.7 \%$, respectively. The axial ratio bandwidth and transmission conversion efficiency at operated frequency bands $(f 1)$ and $(f 2)$ are comparatively better than reported work [25]. The RHCP waves are obtained at the operated frequency bands from $10.48 \mathrm{GHz}$ to $10.78 \mathrm{GHz}$ and $10.91 \mathrm{GHz}$ to $11.18 \mathrm{GHz}$.

2.2. Multiband Circular Polarizer. Subsequently, the concept of dual-band circular polarizer is extended to compose multiband circular polarizer by implementing $2 \times 2$ array. Figure 3 depicts the view of proposed polarizer which is constructed of $2 \times 2$ array of printed metallic strips on both sides of the substrate. The array occupies an overall area of $30 \mathrm{~mm} \times 30 \mathrm{~mm}$. The bottom tilted metallic strips' pattern is formed by rotating an enantiomeric form of the upper slanted strips' pattern by $90^{\circ}$.

In simulation process, the periodic boundary conditions are used to $x$ and $y$ direction and absorption boundary conditions are applied along the perpendicular to the direction of EM wave propagation. The copolarization $T_{x x}$ and crosspolarization $T_{x y}$ transmission are achieved when structure is illuminated by $x$-polarized wave along $+z$ direction. The transmission coefficients of the circularly polarized wave can be calculate by expression of $t_{ \pm}=1 / \sqrt{ } 2\left(t_{x x} \pm i t_{x y}\right)$ for normal incident of $x$-polarized wave, where the subscript " + " represents the RHCP wave, "-" indicates the LHCP wave, $1 / \sqrt{ } 2$ is denoted for power normalization, and $t$ represents the transmission wave.

To elucidate the physical origin of strong circular dichroism and optical activity of our design, the surface current distribution can be observed on top and bottom layers at 


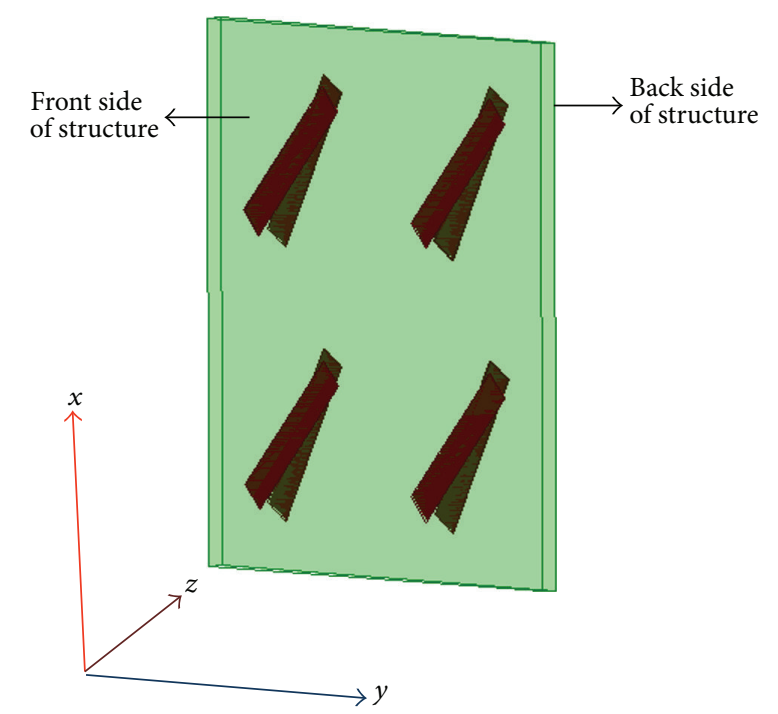

FIGURE 3: The figure presents the multiband circular polarizer based on $2 \times 2$ periodic metallic strips array.

the resonant frequencies, as depicted in Figure 4. The solid (dash) line arrows indicate the front (back) surface current distribution and electric field direction on the top and bottom strips. The currents on the top and bottom layers are in opposite direction at $10.24 \mathrm{GHz}, 10.54 \mathrm{GHz}, 11.09 \mathrm{GHz}$, and $11.14 \mathrm{GHz}$, corresponding to an asymmetric resonance mode.

From Figure 4, it can be observed that the surface currents' directions on the top and bottom strips are antiparallel at all distinct resonant frequencies, which show the effective current loops. It is realized that the magnetic vectors induced by current loops on the surface of the strips are not perpendicular to the electric vectors of the incident wave; therefore, the cross-coupling behaviour between electric and magnetic fields is realized at four resonant frequencies. Similarly, the surface current distribution at the end portion of the strips is very week because the magnetic field is parallel to the $y$ direction and electric field along $x$ direction, resulting in the transmitted cross-polarized wave.

Figure 4 represents the direction of currents which are totally antiphase between two strips and attributes the giant circular polarization conversion at distinct frequencies. The incident $x$-polarized wave excites the current loops in metallic strips which produces the magnetic dipole moment. In addition, the electric and magnetic responses exist not only on the surface of each strip but also between the strips due to the strong coupling effect [26-28].

For the experimental purpose, a prototype sample of the proposed circular polarizer is fabricated to verify its performance. The fabrication is carried out through conventional circuit board technology with the same designed model parameters as the simulation structure. Figure 5 depicts the photograph of the fabricated sample of multiband circular polarizer.

The measurement is carried out to validate the simulation results of polarizer structure in microwave anechoic chamber by free space method. The fabricated sample is perpendicular placed in the middle between two linearly polarized horn antennas; one is working as transmitter and the other as receiver. The distance between fabricated sample and antenna is equal to the numerical simulation approximately. The antennas are connected to two ports of vector network analyzer of Agilent N5230C, which produce EM microwave in the range of $8-12 \mathrm{GHz}$.

It can be possible to obtain the corresponding all vector components of the EM wave by changing the orientations of the horn antennas. For the measurement of cross-polar transmission, one horn antenna is placed vertically while the other is placed horizontally in order of transmitterreceiver alignment. The measured copolar reference is used for the cross-polar measurements. The raw data is shown in oscillating behaviour; therefore, a data processing procedure similar to that in [9] is used to remove oscillations behaviour from data.

Figure 6(a) shows the simulated and measured transmission coefficients of copolarization $T_{x x}$. The transmission coefficients of $T_{x x}$ are $10.06 \mathrm{~dB}$ and $9.92 \mathrm{~dB}(f 1=10.24 \mathrm{GHz})$, $9.70 \mathrm{~dB}$ and $9.93 \mathrm{~dB}(f 2=10.54 \mathrm{GHz}), 10.76 \mathrm{~dB}$ and $10.46 \mathrm{~dB}(f 3=11.09 \mathrm{GHz})$, and $10.37 \mathrm{~dB}$ and $10.24 \mathrm{~dB}$ $(f 4=11.14 \mathrm{GHz})$, respectively. Meanwhile, the simulated and measured transmission coefficients of $T_{x y}$ are obtained, $8.17 \mathrm{~dB}$ and $8.15 \mathrm{~dB}(f 1), 8.60 \mathrm{~dB}$ and $8.95 \mathrm{~dB}(f 2), 10.50 \mathrm{~dB}$ and $10.52 \mathrm{~dB}(f 3)$, and $10.22 \mathrm{~dB}$ and $10.25 \mathrm{~dB}(f 4)$, as denoted in Figure 6(b). In this proposed design, the giant circular dichroism and convergence of transmitted wave for multiband circular polarizer are valid for $x$-polarization incident wave propagation along $+z$ direction. The axial ratio between transmitted waves is shown in Figure 6(c). The value of axial ratios is $1.24(1.29)$ at $(f 1), 1.1(1.2)$ at $(f 2), 1.02(1.0)$ at $(f 3)$, and $1.0(0.99)$ at $(f 4)$, respectively.

In Figure 6(d), the simulated and measured results of phase differences are to be $90.28^{\circ}$ and $95.24^{\circ}(f 1),-243.43^{\circ}$ and $-240.16^{\circ}(f 2), 132.84^{\circ}$ and $133.05^{\circ}(f 3)$, and $-225.12^{\circ}$ and $-225.86^{\circ}(f 4)$, respectively. Figure $6(\mathrm{c})$ presents the axial ratio bandwidths at the four different resonances ranging from $8 \mathrm{GHz}$ to $12 \mathrm{GHz}$, which are broaden at $10.08 \mathrm{GHz}-10.70 \mathrm{GHz}$, $\mathrm{BW}=5.16 \%$ and $10.78 \mathrm{GHz}-11.14 \mathrm{GHz}, \mathrm{BW}=3.0 \%$, respectively. The axial ratio bandwidth is extended at operated frequency bands which is comparatively better than reported work [14]. In previous work, the results were demonstrated compared to the RHCP wave and LHCP wave is achieved at two distinct frequencies $f 1$ and $f 2$ [25]. In this research, the proposed multiband structure achieves RHCP wave from 10.08 $\mathrm{GHz}$ to $10.53 \mathrm{GHz}$ and $10.78 \mathrm{GHz}$ to $11.12 \mathrm{GHz}$, whereas LHCP wave is achieved from $10.54 \mathrm{GHz}$ to $10.70 \mathrm{GHz}$ and $11.13 \mathrm{GHz}$ to $11.14 \mathrm{GHz}$, respectively. It is shown that the accumulative axial ratio bandwidth of $8.16 \%$ is obtained for the circular polarization ranging from $8 \mathrm{GHz}$ to $12 \mathrm{GHz}$.

It is expressed that $T_{x x}=E_{x}^{t} / E_{x}^{i}$ and $T_{x y}=E_{y}^{t} / E_{x}^{i}$. In these expressions, the first subscript indicates the output polarized wave and second subscript represents the input signal polarizations, respectively. For instance, $T_{x y}=$ $E_{y}^{t} / E_{x}^{i}$, where $E_{x}^{i}$ is the applied as input $x$-polarized wave along $+z$ direction. If the cross-polarization is lower than copolarization $T_{x y}<T_{x x}$, it means that transmitted waves are 


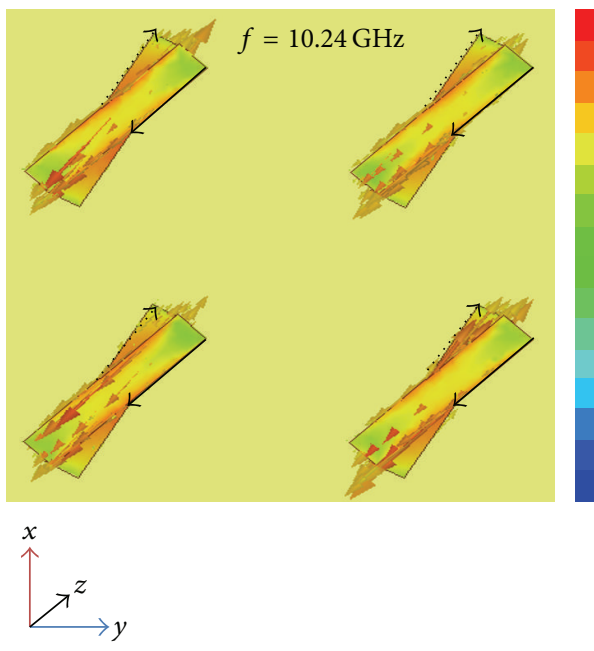

(a)
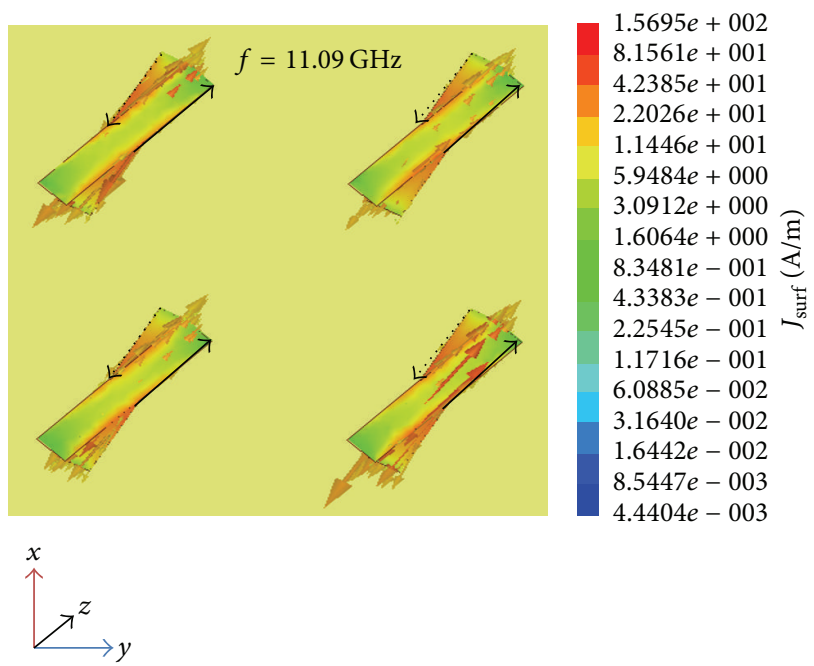
$8.1561 e+001$

$4.2385 e+001$

$2.2026 e+001$

$1.1446 e+001$

$5.9484 e+000$

$3.0912 e+000$

$1.6064 e+000$.

$8.3481 e-001 \longleftarrow$

$4.3383 e-001$

$2.2545 e-001 \stackrel{-}{-}$

$1.1716 e-001$

$6.0885 e-002$

$3.1640 e-002$

$1.6442 e-002$

$8.5447 e-003$

$4.4404 e-003$
$1.5695 e+002$

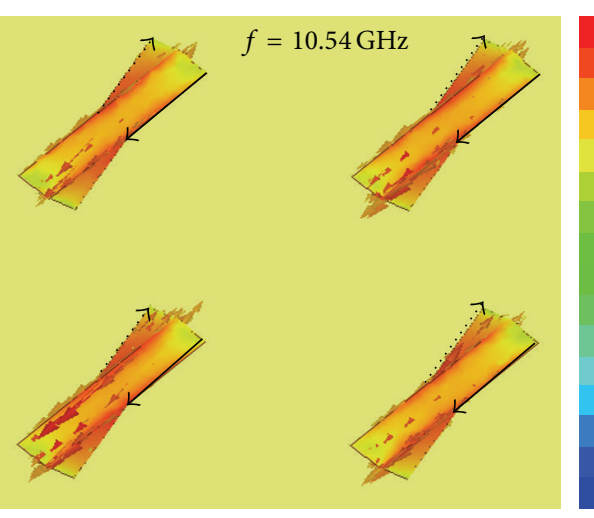

$1.5695 e+002$

$8.1561 e+001$

$4.2385 e+001$

$2.2026 e+001$

$1.1446 e+001$

$5.9484 e+000$

$3.0912 e+000$

$1.6064 e+000$

$8.3481 e-001 \longleftarrow$

$4.3383 e-001$

$2.2545 e-001-$

$1.1716 e-001$

$6.0885 e-002$

$3.1640 e-002$

$1.6442 e-002$

$8.5447 e-003$

$4.4404 e-003$

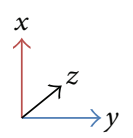

(b)

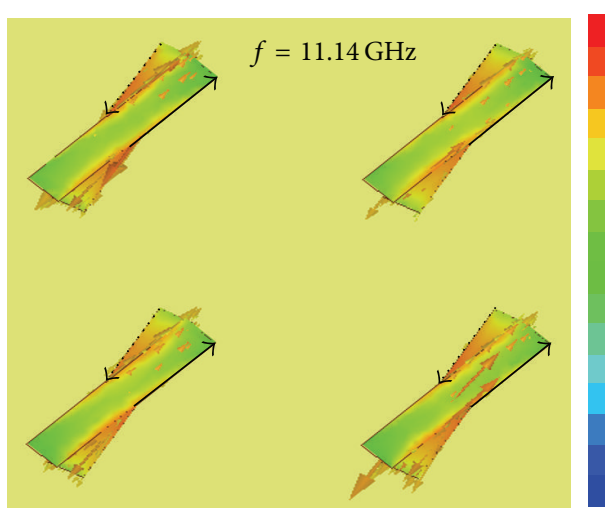

$1.5695 e+002$

$8.1561 e+001$

$4.2385 e+001$

$2.2026 e+001$

$1.1446 e+001$

$5.9484 e+000$

$3.0912 e+000$

$1.6064 e+000$ ह

$8.3481 e-001 \unlhd$

$4.3383 e-001$

$2.2545 e-001-$

$1.1716 e-001$

$6.0885 e-002$

$3.1640 e-002$

$1.6442 e-002$

$8.5447 e-003$

$4.4404 e-003$

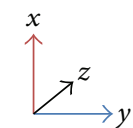

(c)

(d)

Figure 4: The figure shows the distributions of surface currents on top and bottom strips under the incident field with $E$ in the $x$ direction propagating along $+z$ direction at (a) $f=10.24 \mathrm{GHz}$, (b) $f=10.54 \mathrm{GHz}$, (c) $f=11.09 \mathrm{GHz}$, and (d) $f=11.14 \mathrm{GHz}$.
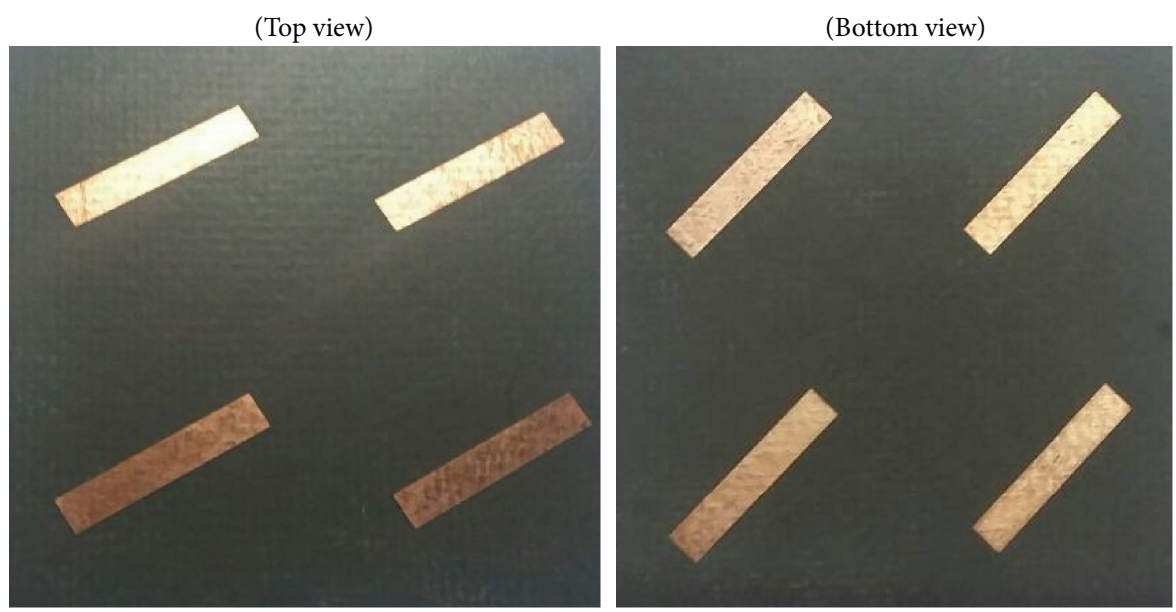

FIGURE 5: The figure shows the photographs of the fabricated sample. 


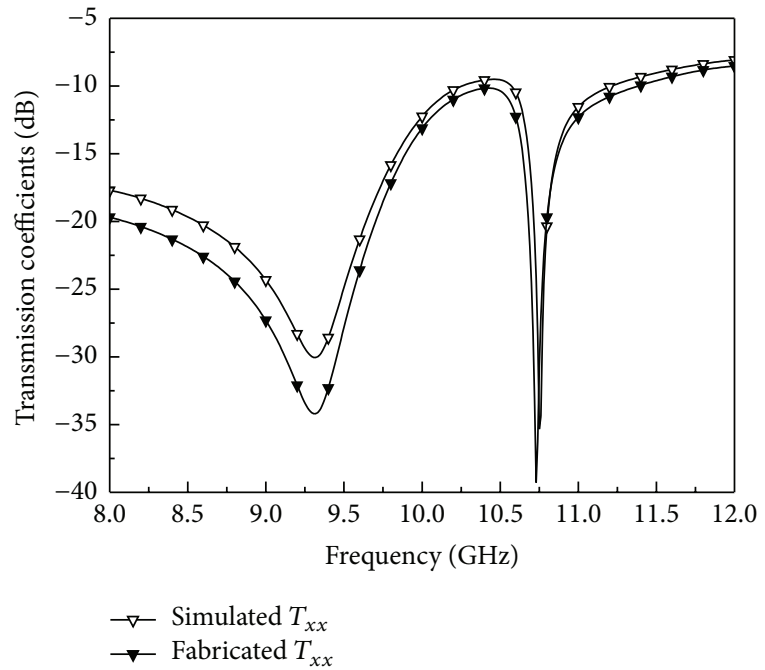

(a)

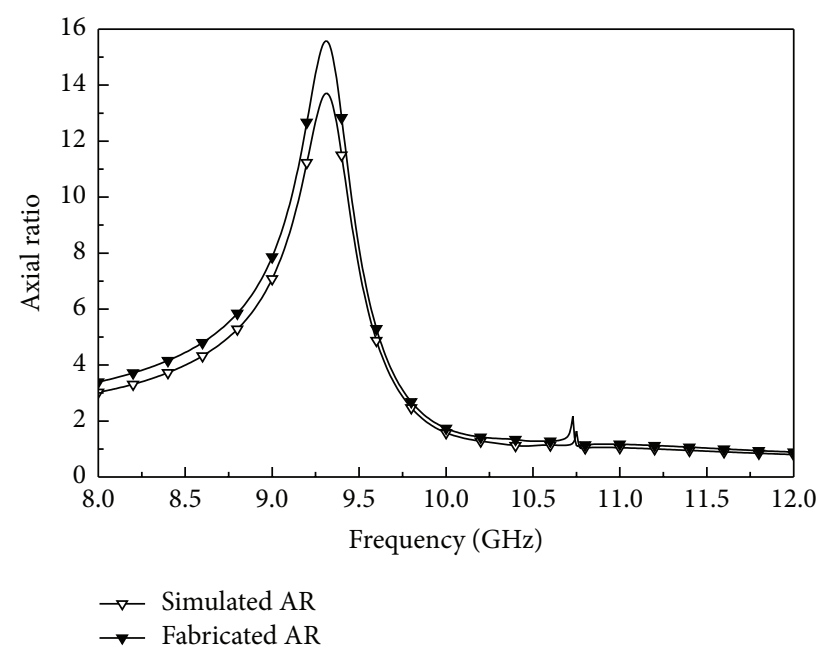

(c)

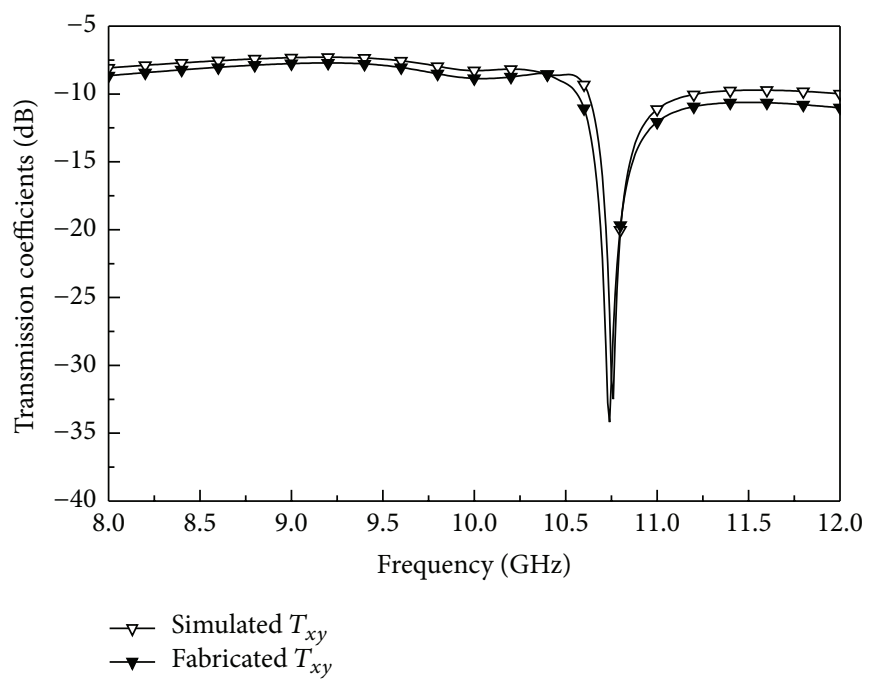

(b)

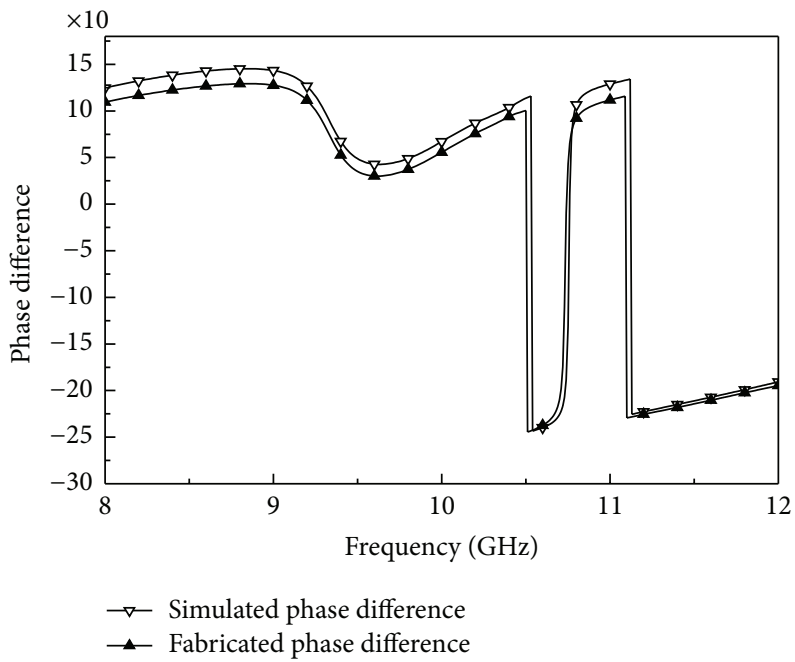

(d)

FIGURE 6: The figure shows the simulation and experimental results for multiband circular polarizer. (a) represents the transmission coefficients of $T_{x x}$ versus frequency for normal incident wave at $\theta=0^{\circ}$, (b) indicates the transmission coefficients of $T_{x y}$ versus frequency for normal incident wave at $\theta=0^{\circ}$, (c) shows the axial ratio versus frequency, and (d) shows the phase difference between transmitted waves versus frequency of designed structure.

elliptic polarization. Therefore, the co- and cross-polarization transmission coefficients should have equal amplitudes and phase difference between them must be $\eta \pi / 2$.

Assume that $T_{x x}=E_{x}^{t} / E_{x}^{i}$ and $T_{x y}=E_{y}^{t} / E_{x}^{i}$ represent the transmittance of $x$-to- $x$ and $x$-to- $y$ polarization conversions. It means that, under the certain coordinate system, the two decomposed components of transmitted linearly polarized waves $E_{x}^{t}$ and $E_{y}^{t}$ from the bottom layer are used as incident polarized waves $E_{x}^{i}$ and $E_{y}^{i}$ for the top layer along the $+z$ direction. As the incidence of $E_{x}^{i}$ and $E_{y}^{i}$ as the excitation source, the two more pairs of orthogonal vector components can be generated at the other side of top layer which realizes "fission yields vector components of electromagnetic waves."

It is assumed that four transmitted orthogonal vector components converge to each other and form strong circular dichroism at the end of structure. The physics of operating principle based on the fission transmission of electromagnetic waves which realizes the incident wave decomposed into two transmitted waves and output decomposed waves impinge on the upper layer of structure to yield four transmitted waves. In addition, the sequence of incident and transmission wave can produce more transmission wave and sustain the chain transmission of EM waves by using doublelayer structure.

\section{Conclusion}

In summary, the proposed structure is comprehensively characterized. The various designed structures demonstrated the giant circular dichroism with different configurations as reported in this work. Firstly, we introduce the novel 
concept of "fission transmission of EM waves" and its physics to realize strong circular dichroism at distinct resonant frequencies. The simulated and measured results reveal that giant circular dichroism effect with multibands is obtained for circular polarization. The simulated and measured results of our research exhibit good correspondence. It is assumed that the fission transmission of EM waves can be realized by employing multilayers periodic layers printed on both sides of substrate. The proposed circular polarizer has good circular polarization efficiency and high transmission efficiency which makes it potentially useful for many practical systems. The designed model of polarizers is very simple and can be easily fabricated. Moreover, it is possible to enhance the bandwidth performance by changing some geometric parameters of the polarizer, such as thickness of substrate, size, and orientation of strips and using multilayers to obtain giant circular dichroism.

\section{Competing Interests}

The authors declared that they have no competing interests.

\section{Authors' Contributions}

Mr. Farman Ali Mangi, Mr. Shaoqiu Xiao, and Ghulam Ali Mallah proposed this idea and constructed methods for this research and Mr. Deedar Ali Jamro worked on the designed simulation models and collected the literature review. A proof of concepts and determination of reported designs were completed by Mr. Imran Memon and Miss Ghulam Fatima Kakepoto. All authors read and approved the final paper.

\section{Acknowledgments}

This work was supported in part by National Natural Science Foundation of China under Grants 61271028, 61271027, and 61101039, in part by Fok Ying Tong Education Foundation under Grant 131107, and in part by Research Fund for the Doctoral Program of Higher Education of China under Grant 20130185110024.

\section{References}

[1] J. Kaschke, J. K. Gansel, and M. Wegener, "On metamaterial circular polarizers based on metal N-helices," Optics Express, vol. 20, no. 23, pp. 26012-26020, 2012.

[2] Y. F. Li, J. Zhang, S. Qu et al., "Wideband selective polarization conversion mediated by three-dimensional metamaterials," Journal of Applied Physics, vol. 115, no. 23, Article ID 234506, 2014.

[3] X. J. Huang, D. Yang, and H. L. Yang, "Multiple-band reflective polarization converter using U-shaped metamaterial," Journal of Applied Physics, vol. 115, no. 10, Article ID 103505, 2014.

[4] Y. Huang, Y. Zhou, and S.-T. Wu, "Broadband circular polarizer using stacked chiral polymer films," Optics Express, vol. 15, no. 10, pp. 6414-6419, 2007.
[5] H.-X. Xu, G.-M. Wang, M. Q. Qi, T. Cai, and T. J. Cui, "Compact dual-band circular polarizer using twisted Hilbertshaped chiral metamaterial," Optics Express, vol. 21, no. 21, pp. 24912-24921, 2013.

[6] M. Mutlu, A. E. Akosman, A. E. Serebryannikov, and E. Ozbay, "Asymmetric chiral metamaterial circular polarizer based on four U-shaped split ring resonators," Optics Letters, vol. 36, no. 9, pp. 1653-1655, 2011.

[7] S. Yan and G. A. E. Vandenbosch, "Compact circular polarizer based on chiral twisted double split-ring resonator," Applied Physics Letters, vol. 102, no. 10, Article ID 103503, 2013.

[8] H. L. Zhu, S. W. Cheung, K. L. Chung, and T. I. Yuk, "Linearto-circular polarization conversion using metasurface," IEEE Transactions on Antennas and Propagation, vol. 61, no. 9, pp. 4615-4623, 2013.

[9] R.-S. Chu and K.-M. Lee, "Analytical model of a multilayered meander-line polarizer plate with normal and oblique planewave incidence," IEEE Transactions on Antennas and Propagation, vol. 35, no. 6, pp. 652-661, 1987.

[10] M. Euler, V. Fusco, R. Cahill, and R. Dickie, "Comparison of frequency-selective screen-based linear to circular splitring polarisation convertors," IET Microwaves, Antennas and Propagation, vol. 4, no. 11, pp. 1764-1772, 2010.

[11] S.-W. Wang, C.-H. Chien, C.-L. Wang, and R.-B. Wu, "A circular polarizer designed with a dielectric septum loading," IEEE Transactions on Microwave Theory and Techniques, vol. 52, no. 7, pp. 1719-1723, 2004.

[12] M. Mutlu, A. E. Akosman, and E. Ozbay, "Broadband circular polarizer based on high-contrast gratings," Optics Letters, vol. 37, no. 11, pp. 2094-2096, 2012.

[13] X. Ma, C. Huang, M. Pu et al., "Dual-band asymmetry chiral metamaterial based on planar spiral structure," Applied Physics Letters, vol. 101, Article ID 161901, 2012.

[14] X. Ma, C. Huang, M. Pu, C. Hu, Q. Feng, and X. Luo, "Multi-band circular polarizer using planar spiral metamaterial structure," Optics Express, vol. 20, no. 14, pp. 16050-16058, 2012.

[15] T. Cao and M. J. Cryan, "Enhancement of circular dichroism by a planar non-chiral magnetic metamaterial," Journal of Optics, vol. 14, no. 8, Article ID 085101, 2012.

[16] Y. Zhao, M. A. Belkin, and A. Alù, "Twisted optical metamaterials for planarized ultrathin broadband circular polarizers," Nature Communications, vol. 3, article 870, 2012.

[17] Y. Cheng, Y. Nie, L. Wu, and R. Gong, "Giant circular dichroism and negative refractive index of chiral metamaterial based on split-ring resonators," Progress in Electromagnetics Research, vol. 138, pp. 421-432, 2013.

[18] F. Dincer, C. Sabah, M. Karaaslan, E. Unal, M. Bakir, and U. Erdiven, "Asymmetric transmission of linearly polarized waves and dynamically wave rotation using chiral metamaterial," Progress in Electromagnetics Research, vol. 140, pp. 227-239, 2013.

[19] J. Li, F.-Q. Yang, and J.-F. Dong, "Design and simulation of Lshaped chiral negative refractive index structure," Progress in Electromagnetics Research, vol. 116, pp. 395-408, 2011.

[20] K. Song, X. P. Zhao, Q. H. Fu, Y. H. Liu, and W. R. Zhu, "Wideangle 90॰-polarization rotator using chiral metamaterial with negative refractive index," Journal of Electromagnetic Waves and Applications, vol. 26, no. 14-15, pp. 1967-1976, 2012. 
[21] Y. Z. Cheng, Y. Nie, and R. Z. Gong, "Giant optical activity and negative refrac-tive index using complementary U-shaped structure assembly," Progress In Electromagnetics Research M, vol. 25, pp. 239-253, 2012.

[22] M. Mutlu, A. E. Akosman, A. E. Serebryannikov, and E. Ozbay, "Asymmetric transmission of linearly polarized waves and polarization angle dependent wave rotation using a chiral metamaterial," Optics Express, vol. 19, no. 15, pp. 14290-14299, 2011.

[23] X. Xiong, W.-H. Sun, Y.-J. Bao et al., "Construction of a chiral metamaterial with a U-shaped resonator assembly," Physical Review B, vol. 81, no. 7, Article ID 075119, 6 pages, 2010.

[24] Z. Li, R. Zhao, T. Koschny et al., "Chiral metamaterials with negative refractive index based on four ' $U$ ' split ring resonators," Applied Physics Letters, vol. 97, no. 8, Article ID 081901, 2010.

[25] H.-X. Xu, G.-M. Wang, M. Q. Qi, and T. Cai, "Dual-band circular polarizer and asymmetric spectrum filter using ultrathin compact chiral metamaterial," Progress in Electromagnetics Research, vol. 143, pp. 243-261, 2013.

[26] Y. Ye and S. He, " $90^{\circ}$ Polarization rotator using a bilayered chiral metamaterial with giant optical activity," Applied Physics Letters, vol. 96, no. 20, Article ID 203501, 2010.

[27] C. Huang, X. Ma, M. Pu, G. Yi, Y. Wang, and X. Luo, "Dualband $90^{\circ}$ polarization rotator using twisted split ring resonators array," Optics Communications, vol. 291, pp. 345-348, 2013.

[28] N. Liu and H. Giessen, "Three-dimensional optical metamaterials as model systems for longitudinal and transverse magnetic coupling," Optics Express, vol. 16, no. 26, pp. 21233-21238, 2008. 


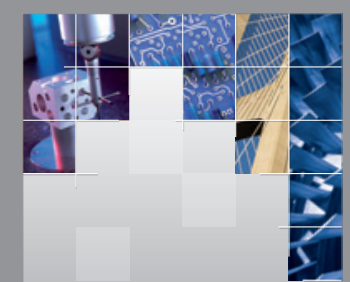

\section{Enfincering}
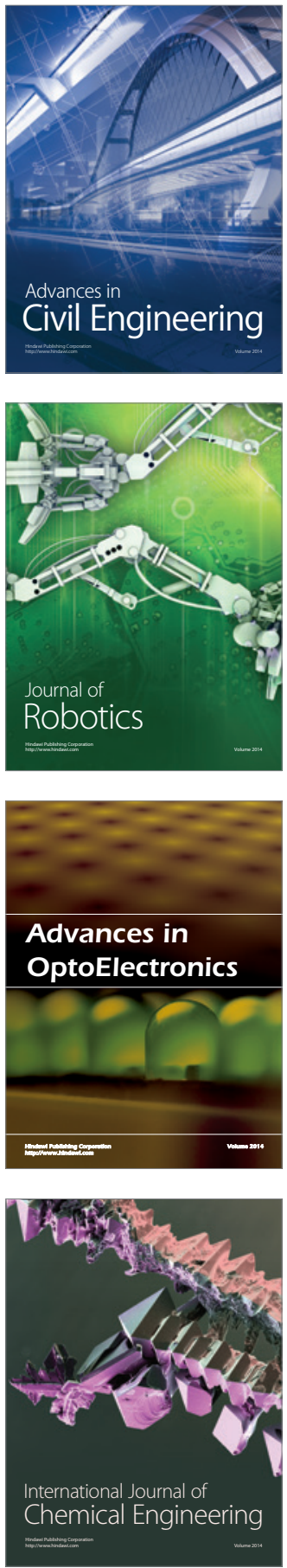

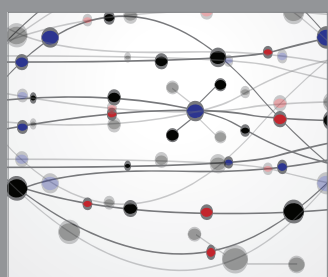

The Scientific World Journal

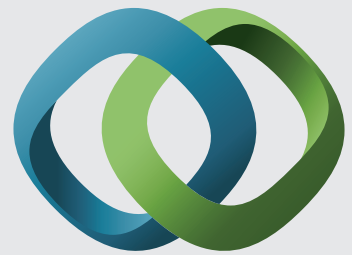

\section{Hindawi}

Submit your manuscripts at

http://www.hindawi.com
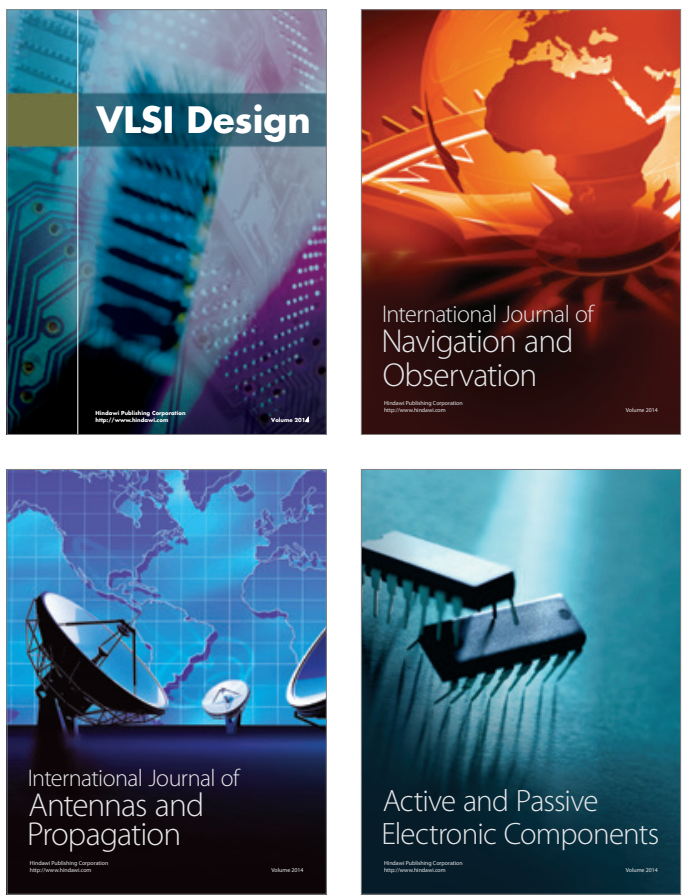
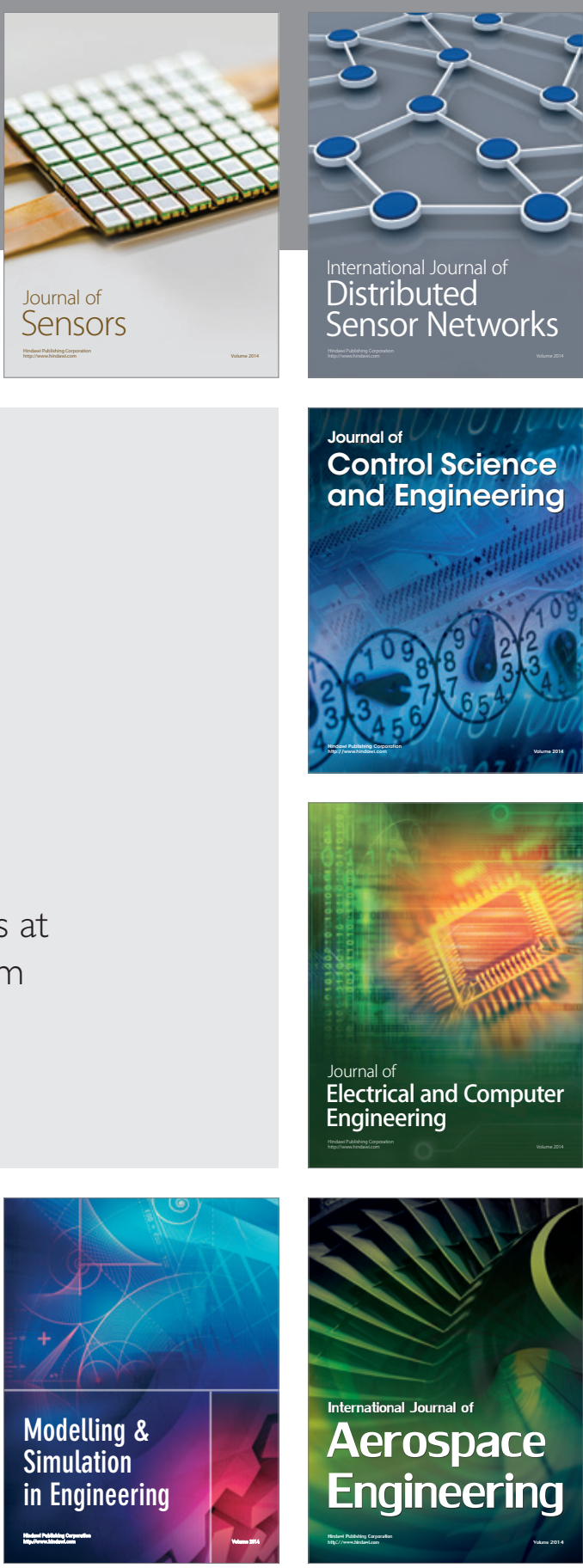

International Journal of

Distributed

Sensor Networks

Journal of

Control Science

and Engineering
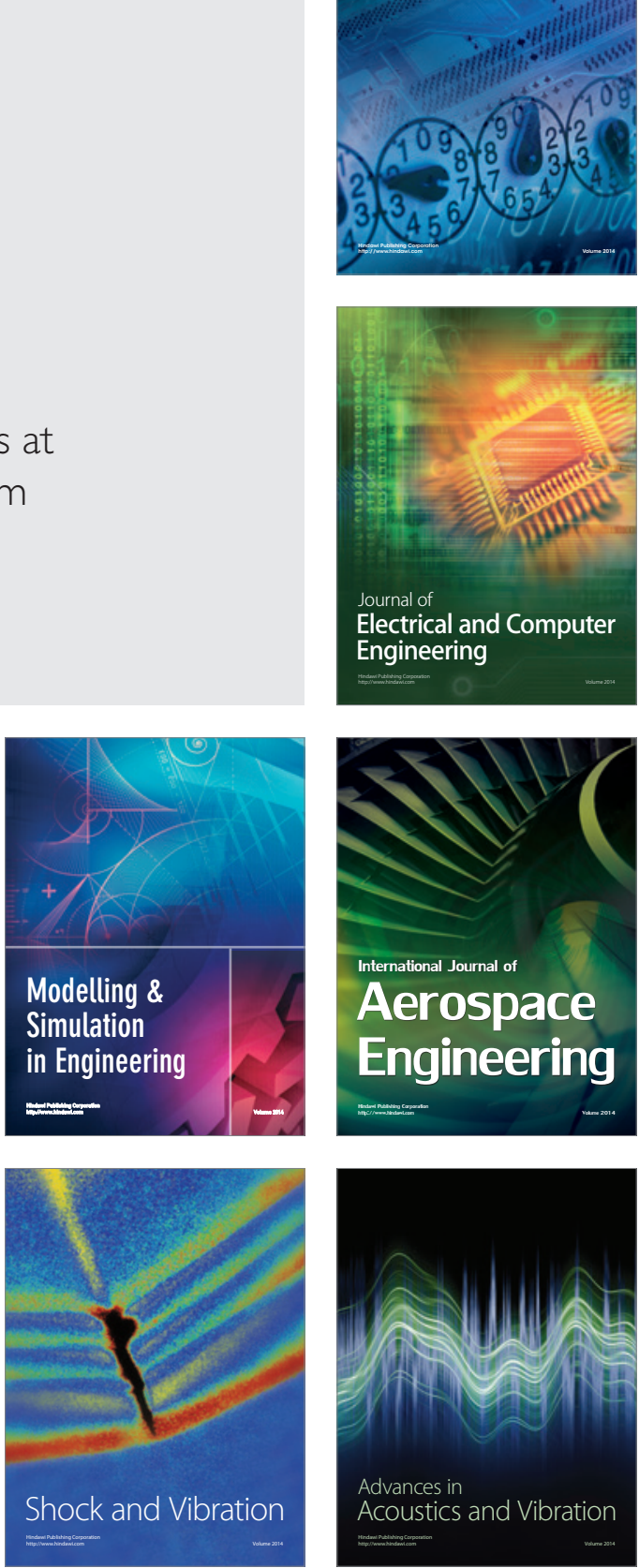